\section{Lattice Compression Increases the Activation Barrier for Phase Segregation in Mixed-Halide Perovskites}

\author{
Loreta A. Muscarella, Eline M. Hutter, Francesca Wittmann, Young Won Woo, Young-Kwang Jung, \\ Lucie McGovern, Jan Versluis, Aron Walsh, Huib J. Bakker, and Bruno Ehrler*
}

Cite This: ACS Energy Lett. 2020, 5, 3152-3158

Read Online

ACCESS

|lll Metrics \& More

Article Recommendations

Supporting Information

ABSTRACT: The bandgap tunability of mixed-halide perovskites makes them promising candidates for light-emitting diodes and tandem solar cells. However, illuminating mixed-halide perovskites results in the formation of segregated phases enriched in a single halide. This segregation occurs through ion migration, which is also observed in single-halide compositions, and whose control is thus essential to enhance the lifetime and stability. Using pressuredependent transient absorption spectroscopy, we find that the formation rates of both iodide- and bromide-rich phases in $\operatorname{MAPb}\left(\mathrm{Br}_{x} \mathrm{I}_{1-x}\right)_{3}$ reduce by 2 orders of magnitude on increasing the pressure to $0.3 \mathrm{GPa}$. We explain this reduction from a compression-induced increase of the activation energy for halide

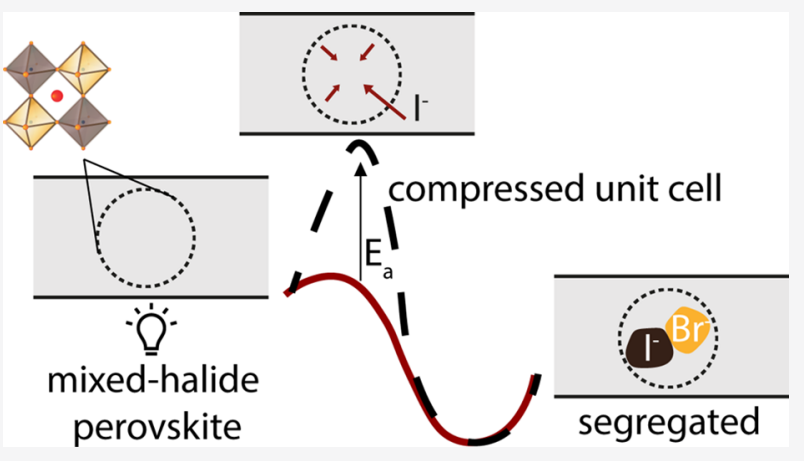
migration, which is supported by first-principle calculations. A similar mechanism occurs when the unit cell volume is reduced by incorporating a smaller cation. These findings reveal that stability with respect to halide segregation can be achieved either physically through compressive stress or chemically through compositional engineering.

$\mathrm{H}$ alide perovskite semiconductors have recently gathered significant attention due to their excellent optoelectronic properties combined with a low-cost and simple fabrication method. In addition, the bandgap of these perovskites can easily be tuned by mixing halides in different ratios, ${ }^{1}$ making them promising candidates for lightemitting diodes (LEDs) ${ }^{2,3}$ and tandem solar cells. ${ }^{4}$ However, methylammonium(MA)-based mixed-halide perovskites, $\operatorname{MAPb}\left(\mathrm{Br}_{x} \mathrm{I}_{1-x}\right)_{3}$, lack long-term bandgap stability under standard solar cell operating conditions. ${ }^{5}$ Compositions with $x>0.20$ have been shown to suffer from halide segregation ${ }^{6}$ during continuous light exposure ${ }^{7}$ or applied bias. ${ }^{8}$ As a result, low- and high-bandgap phases are formed, corresponding to iodide-rich and bromide-rich domains, respectively. ${ }^{6}$ Halide migration affects the homogeneity of the bandgap required for many applications such as lighting and displays, and photogenerated charges transfer into the low-bandgap iodide-rich domains, where they recombine.

Phase segregation of mixed-halide perovskites involves the migration of halide ions. In fact, even single-halide perovskite systems (e.g., $\mathrm{MAPbI}_{3}$ ) suffer from ion migration,, ${ }^{9,10}$ and this process has been identified as one of the main drivers for degradation. ${ }^{11}$ The activation energies reported for halide migration in single-halide systems ${ }^{10,12}$ and in mixed-halide systems $^{6,12}$ are comparable, suggesting a similar transport mechanism in both cases. Therefore, both single- and mixedhalide perovskites would benefit from increasing the activation energies for ion migration, as this would slow down the degradation rate and hence increase the lifetime of the corresponding device. Phase segregation has been shown to depend on various factors such as light intensity, ${ }^{12-14}$ duty cycle, ${ }^{15}$ and film quality and thickness. ${ }^{14,16}$ Reducing the halide vacancy concentrations ${ }^{17}$ and passivating electron traps, ${ }^{18}$ whose electric field has been proposed to initiate halide migration by accumulating holes, have been suggested as ways to reduce the rate of phase segregation. The same result has also been achieved by partial replacement of the organic cation with the smaller $\mathrm{Cs}^{+}$in the mixed perovskite. ${ }^{19-22}$

Received: July 9, 2020

Accepted: September 1, 2020

Published: September 1, 2020 
a)

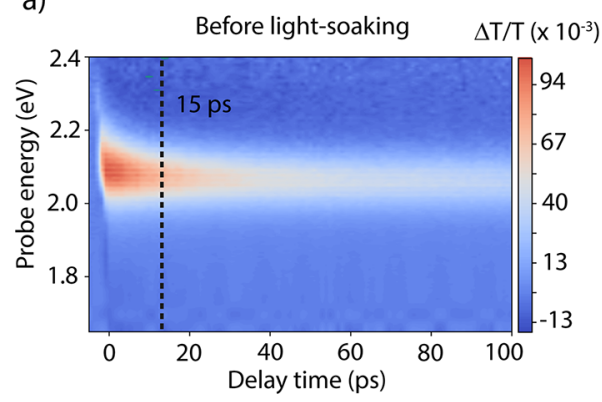

b)

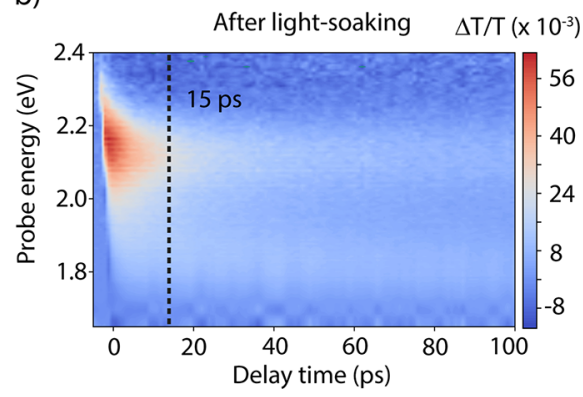

Figure 1. (a) $\Delta T / T$ as a function of the probe energy and delay time between the pump and probe pulse for $\mathrm{MAPb}\left(\mathrm{Br}_{0.5} \mathrm{I}_{0.5}\right)_{3}$ before lightsoaking, where only the bleach from the mixed-halide composition $(x=0.5)$ is observed, and (b) after 20 min of light-soaking with a CW laser, where a bleach from two phases peaking at 1.85 and $2.15 \mathrm{eV}$ is observed, indicative of halide segregation. The measurements are performed at ambient pressure. The dashed line indicates the delay time chosen to study the kinetics of phase segregation.

a)

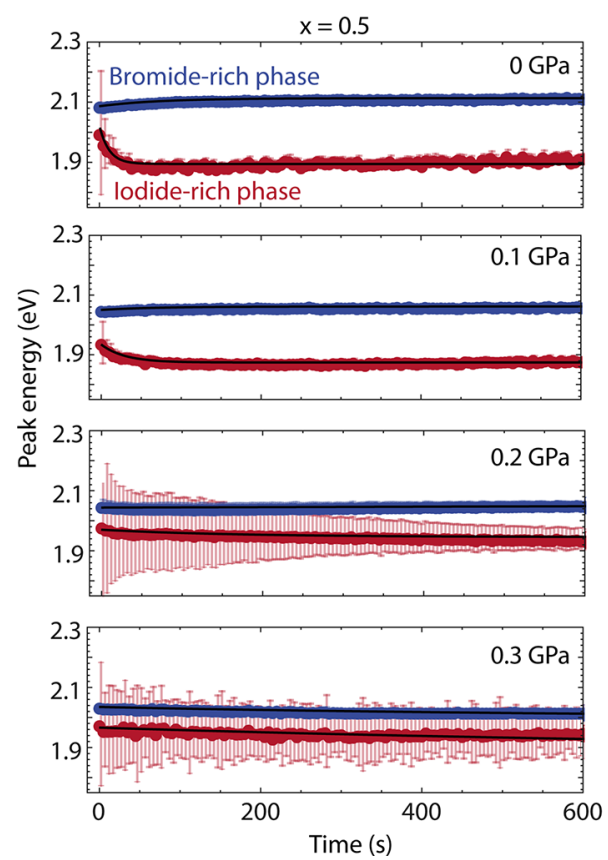

b)
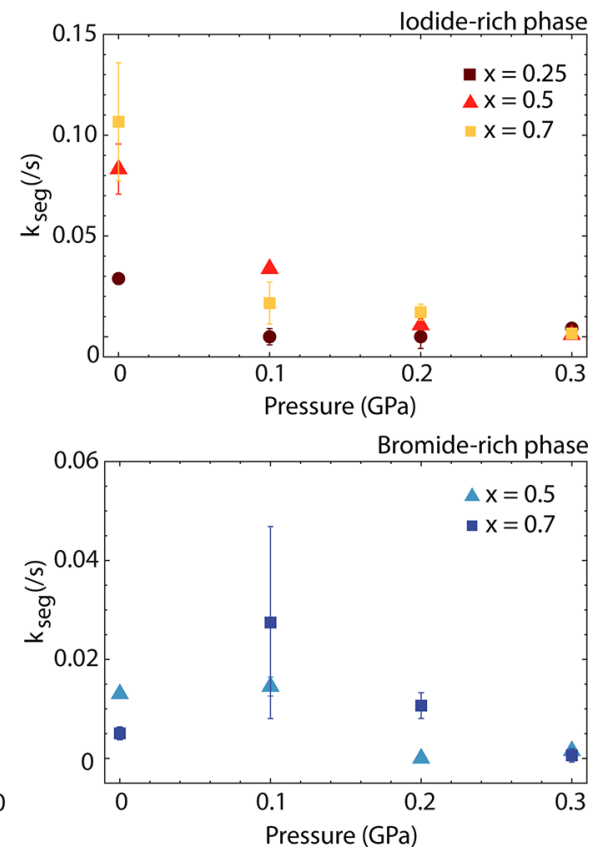

Figure 2. (a) Dynamic evolution of the low-energy iodide-rich phase (red) and high-energy bromide-rich phase (blue) measured by pressure-dependent TAS during light-soaking with a CW laser from ambient pressure to $0.3 \mathrm{GPa}$. (b) Segregation rates for the iodide- and bromide-rich phases as a function of pressure for all the $\operatorname{MAPb}\left(\mathrm{Br}_{x} \mathrm{I}_{1-x}\right)_{3}$ compositions $(x=0.25, x=0.5$, and $x=0.7)$.

Here, we investigate the dependence of the kinetics of phase segregation on structural parameters in mixed-halide perovskite $\operatorname{MAPb}\left(\mathrm{Br}_{x} \mathrm{I}_{1-x}\right)_{3}$ by using pressure-dependent transient absorption spectroscopy. The measurements are performed at hydrostatic pressures ranging from 0 (ambient pressure) to $0.3 \mathrm{GPa}$ using an additional light beam to induce phase segregation. Previously, we established that pressure changes the thermodynamic landscape for phase segregation by finding the final mixing ratio of the segregated phases to be closer to the one of the mixed phase upon increasing pressure. ${ }^{23}$ Here, we find that phase segregation is also substantially slower at high pressure for all $\mathrm{MAPb}\left(\mathrm{Br}_{x} \mathrm{I}_{1-x}\right)_{3}$ mixing ratios $(x=0.25$, 0.5 , and 0.7$)$. We explain this slowing down from an increase of the activation energy $\left(E_{a}\right.$, in light) for halide migration upon compression of the unit cell volume. Theoretical calculations corroborate this interpretation, revealing an increase in the activation energy $\left(E_{\mathrm{a}}\right)$ required for a halide species to diffuse into a vacancy under pressure. In addition, we find that the reduction of the unit cell volume at ambient pressure by partial replacement of the $\mathrm{MA}^{+}$cation with the smaller $\mathrm{Cs}^{+}$, e.g., $\mathrm{MA}_{0.7} \mathrm{Cs}_{0.3} \mathrm{~Pb}\left(\mathrm{Br}_{0.5} \mathrm{I}_{0.5}\right)_{3}$ and $\mathrm{CsPb}\left(\mathrm{Br}_{0.5} \mathrm{I}_{0.5}\right)_{3}$, also leads to slower segregation, in a similar manner to the reduced segregation rate of $\mathrm{MAPb}\left(\mathrm{Br}_{0.5} \mathrm{I}_{0.5}\right)_{3}$ under high-pressure conditions. Altogether, these findings suggest that the reduction of the unit cell volume achieved through compositional engineering or physical pressure can be effectively used to delay halide migration by increasing the activation barrier of the migration process.

$\mathrm{MAPb}\left(\mathrm{Br}_{x} \mathrm{I}_{1-x}\right)_{3}$ thin films with $x=0,0.25,0.5,0.7$, and 1 were prepared by spin coating the precursor solutions onto quartz substrates as reported elsewhere. ${ }^{23}$ Transient absorption spectroscopy (TAS) allows us to probe the excited state of $\operatorname{MAPb}\left(\mathrm{Br}_{x} \mathrm{I}_{1-x}\right)_{3}$ by recording absorption spectra of the probed area at each delay time (from 0.05 to $800 \mathrm{ps}$ ) following a pulsed pump excitation $(400 \mathrm{~nm}$, with an excitation density of $\sim 10^{18}$ absorbed photons $/ \mathrm{cm}^{3}$ corrected for the fraction of absorbed photons by each composition). Figure 1a shows a $2 \mathrm{D}$ plot of the transient absorption signal of $\mathrm{MAPb}\left(\mathrm{Br}_{0.5} \mathrm{I}_{0.5}\right)_{3}$ as a 
a)

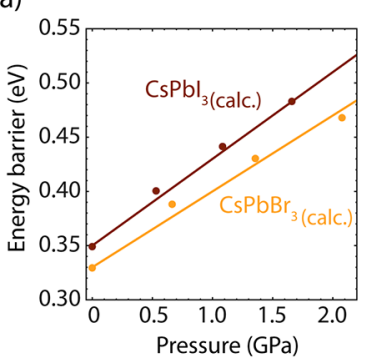

b)

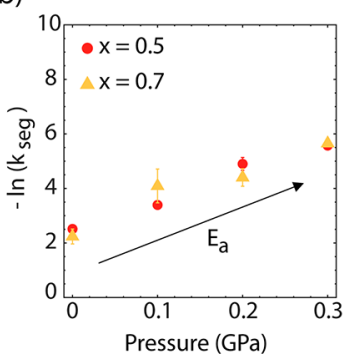

C)

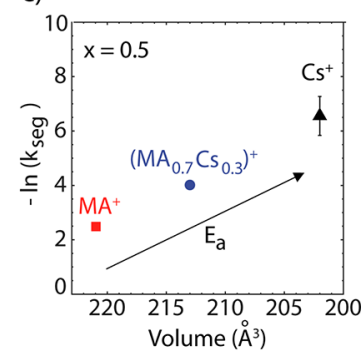

Figure 3. (a) Calculated (DFT/PBEsol) activation energy for iodide and bromide diffusion in $\mathrm{CsPbI}_{3}$ and $\mathrm{CsPbBr}$ as a function of unit cell volume. (b) $-\ln \left(k_{\text {seg }}\right)$, which is proportional to the activation energy for phase segregation, plotted against the external physical pressure applied for $x=0.5$ and $\boldsymbol{x}=0.7$ and (c) against the unit cell volume for $\boldsymbol{x}=\mathbf{0 . 5}$, where the reduction in unit cell volume is achieved via partial replacement of $\mathrm{MA}^{+}$with the smaller $\mathrm{Cs}^{+}$. Here, $-\ln \left(k_{\text {seg }}\right)$ follows the same trend as the high-pressure conditions. We observe that $-\ln \left(k_{\text {seg }}\right)$ follows the same trend as when the pressure is varied.

function of the delay time between the pump and probe and the probe pulse energy. In the absence of an additional light source, $\mathrm{MAPb}\left(\mathrm{Br}_{0.5} \mathrm{I}_{0.5}\right)_{3}$ shows a ground-state bleach at 2.05 $\mathrm{eV}$ in agreement with the absorption onset obtained from steady-state absorption measurements previously reported in Hutter et al. ${ }^{23}$ We then focus a continuous wave (CW) light source $\left(\lambda=405 \mathrm{~nm}, I=2.37 \times 10^{3} \mathrm{~mW} / \mathrm{cm}^{2}\right)$ on the pump spot to induce phase segregation. In Figure $1 \mathrm{~b}$, we show the $2 \mathrm{D}$ transient absorption signal of $\mathrm{MAPb}\left(\mathrm{Br}_{0.5} \mathrm{I}_{0.5}\right)_{3}$ after $20 \mathrm{~min}$ of light-soaking. Now, we observe two bleaching signals, one at lower energy $(1.85 \mathrm{eV})$ and one at higher energy $(2.15 \mathrm{eV})$, that can be assigned to iodide- and bromide-rich domains, respectively. At later times, the bleaching signal from the bromide-rich phase decays, indicating the transfer of photogenerated charges into the iodide-rich phase.

To investigate the kinetics of the phase segregation in $\operatorname{MAPb}\left(\mathrm{Br}_{0.5} \mathrm{I}_{0.5}\right)_{3}$ during light-soaking, we fixed the delay stage position at $15 \mathrm{ps}$, when most of the hot charge carriers induced by the high-energy pump have cooled ${ }^{24}$ to the band edges of the high- and low-energy phases. An important advantage of TAS with respect to time-resolved photoluminescence (TRPL) in this context is the ability to track the excited population from both the iodide- and bromide-rich phases, ${ }^{13}$ whereas TRPL mainly probes the emissive low-energy phase. We recorded transient absorption spectra every second for $20 \mathrm{~min}$. When only the pump excitation is present, we do not observe any phase segregation (see Figure S1). When the mixed-halide perovskite film was subjected to $\mathrm{CW}$ light irradiation at ambient pressure, we observe the appearance of a second peak at lower energy after a few seconds, which subsequently grows in amplitude (see Figure S2). At the same time, the original bleach shifts to higher energy.

Subsequently, we investigated the segregation as a function of compressive stress, which reduces the unit cell size, by performing pressure-dependent transient absorption measurement of the sample inside a hydrostatic pressure cell filled with inert liquid (FC-72, see Experimental Methods). The change in hydrostatic pressure is achieved by increasing the amount of inert liquid through a manual pump, with a resulting pressure ranging from 0 (ambient pressure) to $0.3 \mathrm{GPa}$. The change in unit cell volume calculated by using the bulk moduli ${ }^{23}$ from ambient pressure to $0.3 \mathrm{GPa}$ is about $2.5 \%$ for $x=0.5$ ( 3 and $2.2 \%$ for $x=0.25$ and 0.7 , respectively). The dynamic ingrowth of the iodide- and bromide-rich phases during light-soaking is shown in Figure 2a. As pressure increases, we observe a substantial slowing down of the phase segregation. This observation holds true for all $\mathrm{MAPb}\left(\mathrm{Br}_{x} \mathrm{I}_{1-x}\right)_{3}$ compositions studied, e.g., $x=0.25$ (which barely segregates from $0.1 \mathrm{GPa}$ onward) and $x=0.7$, as reported in Figure S3. We note that at high pressure, the early times bleach can be fitted with a single Gaussian representing one mixed phase (see Figure S4).

To extract the segregation rate as a function of pressure, Gaussian profiles are fit to the ground-state bleaches of the low- and high-energy peak positions in energy, and we monitor the peak energies of these Gaussians as a function of lightsoaking time. All kinetic traces are characterized by a continuous change in the ground-state bleach energy, due to a change in composition, followed by saturation at a certain energy when segregation is complete. As reported in our previous work, ${ }^{23}$ the segregated peaks are closer in energy at high pressure, which is attributed to a change in the terminal $x$ value as a function of the unit cell volume. In addition, we observe that the time at which this final composition is reached changes substantially with pressure. The traces were fitted with monoexponential curves, $A \mathrm{e}^{k_{\mathrm{seg}} t}+c(\mathrm{eV}$, offset $)$, to determine the segregation rate $k_{\text {seg }}$. Our rates at ambient pressure are comparable to previous work calculated under comparable light-soaking conditions. ${ }^{13,25}$

Under pressure, the iodide-rich phase formation rate is greatly decreased. For $x=0.5, k_{\text {seg }}$ decreases from $(0.08 \pm$ $0.01) \mathrm{s}^{-1}$ at ambient pressure to $(0.003 \pm 0.001) \mathrm{s}^{-1}$ at 0.3 $\mathrm{GPa}$, which is a factor of $27 \times$. At $0.3 \mathrm{GPa}$, the difference between the initial and final energies is much smaller than at ambient pressure. ${ }^{23}$ However, still the time constant associated with the evolution toward the terminal $x$-value is much longer, indicating that segregation is substantially slower at $0.3 \mathrm{GPa}$. A similar trend is observed for $x=0.7$ and $x=0.25$, where the segregation rate decreases from $k_{\text {seg }}=(0.1 \pm 0.03) \mathrm{s}^{-1}$ at ambient pressure to $k_{\text {seg }}=(0.003 \pm 0.001) \mathrm{s}^{-1}$ at $0.3 \mathrm{GPa}(33 \times$ reduction $)$ and from $k_{\text {seg }}=(0.028 \pm 0.002) \mathrm{s}^{-1}$ to $k_{\text {seg }}=(0.004$ $\pm 0.001) \mathrm{s}^{-1}(7 \times$ reduction $)$, respectively. For $x=0.25$, there was no detectable change in the bromide-rich peak within our resolution. For the other compositions, the formation rate of the bromide-rich phase is slower and less affected by pressure compared to the iodide-rich phase (Figure 2b). This observation will be discussed in more detail later.

We explain the diminution of the segregation rate upon increasing pressure from an increase of the activation energy associated with the migration of halide ions, consequently delaying the accumulation of ions in the low-energy phase. Density functional theory calculations on the model systems $\mathrm{CsPbI}_{3}$ and $\mathrm{CsPbBr}_{3}$ in Figure 3a show the energy barrier associated with vacancy-assisted halide diffusion. The potential energy surface for ion diffusion is calculated, and the saddle 
point is identified, as a function of applied pressure. The transition state increases in energy as the cell volume decreases (see Figure S5). As a result, the energy barrier increases with pressure for both materials, with a change of $0.134 \mathrm{eV}$ (I) and $0.138 \mathrm{eV}(\mathrm{Br})$ when the pressure is increased from 0 to $2 \mathrm{GPa}$. Over the pressure range from 0 to $0.3 \mathrm{GPa}$, the changes are smaller but can still have a significant impact on the migration rate due to the exponential scaling. For example, a change in the activation energy from $0.35 \mathrm{eV}(0 \mathrm{GPa})$ to $0.38 \mathrm{eV}(0.3$ GPa) as calculated for $\mathrm{CsPbI}_{3}$ results in a $3 \times$ decrease in the vacancy hopping probability at room temperature. These model calculations only consider the effect of pressure on the unit cell volume in the dark. In the real hybrid materials, strain will also impact the dynamics of molecular rotations as well as the distributions of octahedral titling and crystal distortions. In this way, we simulate the correct trend but underestimate with respect to the experimental observations.

To derive the relation between the segregation rate and an effective activation energy for ion migration, we use the definition of the diffusion coefficient ${ }^{26}$ (see Supplementary Note 1 for the full derivation)

$$
k_{\text {migration }}=\frac{q^{2} N \nu_{0} d^{2}}{6 \epsilon_{0} \epsilon_{\text {pero }} k_{\mathrm{B}} T} \exp \left(\frac{\Delta^{\ddagger} S^{\circ}}{k_{\mathrm{B}}}\right) \exp \left(-\frac{E_{\mathrm{a}}}{k_{\mathrm{B}} T}\right)
$$

where $\Delta^{\ddagger} S^{\circ}$ represents the changes in the entropy of a single ion migration step, $k_{\mathrm{B}}$ is the Boltzmann constant, $\nu_{0}$ is the attempt frequency for an ion to hop, $d$ is the ionic hopping distance, $E_{\mathrm{a}}$ is the activation energy, $q$ is the elementary charge, $\epsilon_{\text {pero }}$ is the permittivity of the perovskite, $\epsilon_{0}$ is the permittivity in a vacuum, and $N$ is the doping density. These terms can be approximated (see Supplementary Note 1 for discussion) such that the natural logarithm of the migration rate, which in our experiments correspond to $k_{\text {seg }}$, is proportional to the activation energy for the migration process as follows

$$
-\ln \left(k_{\text {migration }}\right) \propto E_{\mathrm{a}}
$$

For simplicity, we define the experimental $-\ln \left(k_{\text {seg }}\right)$ as the effective activation energy $E_{a}^{*}$. Following this definition, typical activation energies for halide migration in these perovskites are on the order of 100-200 meV (see transient ion drift (TID) measurements below). ${ }^{12,25,27}$ The experimental $E_{a}^{*}$ plotted as a function of physical pressure applied increases by a factor of 3 upon increasing pressure to $0.3 \mathrm{GPa}$ for all the compositions (Figure 3b). Interestingly, we show in Figure $3 c$ that a similar reduction in the phase segregation rate can be achieved by partial or complete replacement of $\mathrm{MA}^{+}$with the smaller cation $\mathrm{Cs}^{+}$instead of applying physical pressure (see Figure S6 for peak energy versus time in the chemically compressed compositions). In the former case, the unit cell volume change measured is $\sim 20 \AA^{3}$, whereas the application of physical pressure leads to a unit cell volume change of $\sim 5 \AA^{3}$ for all the compositions studied. The observation of slower segregation rates suggests that slower ion migration is a phenomenon that is more generally linked to a reduction of the unit cell volume, independent of how this reduction is achieved (see Figure S7 for $E_{a}^{*}$ as a function of unit cell volume change induced by chemical and physical pressure). In earlier work, we found that this generalization is also true for the thermodynamic stability of these mixed-halide perovskites, ${ }^{23}$ pointing toward the importance of the unit cell volume for both kinetic and thermodynamic properties of ion migration. We note here that partial replacement of $\mathrm{MA}^{+}$with the smaller $\mathrm{Cs}^{+}$may result in different defect densities, crystallinities, and strains, which will also affect the absolute segregation rate. In fact, despite the difference in volume change induced by physical pressure $(\sim 5$ $\left.\AA^{3}\right)$ and $\mathrm{MA}^{+}$chemical replacement $\left(\sim 20 \AA^{3}\right)$, the change in activation energy experimentally observed is of the same order of magnitude. This suggests that when the unit cell volume is reduced by $\mathrm{MA}^{+}$replacement, parameters other than the unit cell volume play a role, whereas by applying external physical pressure, we essentially vary only one parameter. However, the similarity in the trends observed in Figure $3 b, c$ indicate that the unit cell volume plays a key factor in the phase segregation rate. As local stoichiometric variations could also play a role in establishing the phase segregation absolute rates, further investigations on single crystals would be useful, as they show less stoichiometric variations compared to thin films. ${ }^{28}$

Ion migration plays a large role in the degradation of perovskite thin films and the effect of strain shimmers through many observations related to perovskite stability. Under tensile strain, the inorganic $\left[\mathrm{PbX}_{6}\right]^{4-}$ framework is distorted, resulting in longer and weaker $\mathrm{Pb}-\mathrm{X}$ bonds and less strongly tilted octahedra. $^{29}$ This strain results in a decreased formation energy for defects and a lower activation energy for ion migration. ${ }^{30} \mathrm{~A}$ source of tensile strain, which lowers the activation energy for ion migration, ${ }^{30-32}$ is the presence of light during lightsoaking, which has been shown to lead to thermal expansion. ${ }^{33}$ We also find a lower activation energy under light exposure than under dark conditions, which we measured using TID on an $x=0.20$ sample, the composition with the highest mixing ratio that does not segregate under light-soaking. We found two negatively charged species migrating with comparable activation energy in dark, namely, $0.15 \pm 0.03$ and $0.14 \pm 0.01$ $\mathrm{eV}$. In the single-halide composition, only one negative species was observed, ${ }^{10}$ so it is likely that the two species found in the mixed-halide compositions represent both iodide and bromide. In light, the effective activation energy of the former species is reduced to $0.09 \pm 0.01 \mathrm{eV}$, whereas the latter remains constant at $0.13 \pm 0.02 \mathrm{eV}$. The decrease in activation energy in light is consistent with the observation that light-induced tensile strain leads to increased ion migration. We note that some of the change in activation energy could be due to heating, but it could not explain the full effect observed. ${ }^{34}$ Since light changes the activation energy of only one of the two halide migration processes, we propose that the process with the lower activation energy in light may be mainly responsible for phase segregation (see Supplementary Note 2 and Figure S10 for TID traces and details on the fitting method).

Our observation that the rate of phase segregation strongly depends on pressure demonstrates the large role that strain plays for ion migration. We hypothesize that pressure-induced compressive stress counteracts the effect of thermal expansion induced by light. Therefore, by stiffening the $\mathrm{Pb}-\mathrm{X}$ bonds, pressure mitigates the reduction in the activation energy for halide migration. A scheme of the proposed mechanism at the microscopic and macroscopic level is shown in Figure $4 a$ and $b$, respectively. A similar reduction of phase segregation can be obtained at ambient pressure by inducing strain via either regulating strain in the perovskite film through chargetransport layers as recently reported by Xue et al. ${ }^{35}$ or through compositional engineering, by partial replacement of $\mathrm{MA}^{+}$with smaller cations. As a consequence, $\mathrm{Cs}^{+}$incorporation further slows down phase segregation in MA-based mixed-halide perovskites. ${ }^{36}$ Ferdani et al. ${ }^{37}$ have also reported increased activation energy for iodide migration by measuring impedance 
a)

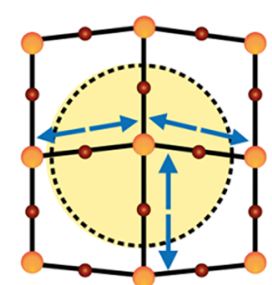

Light-induced strain

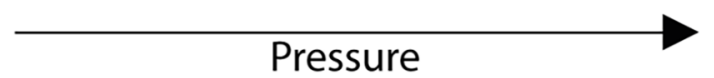

b)

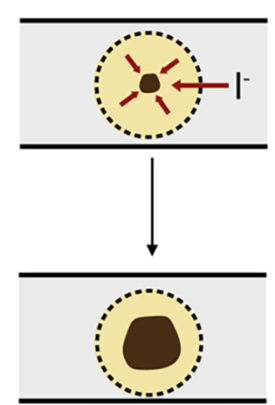

Fast segregation

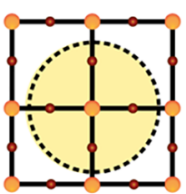

Less strain

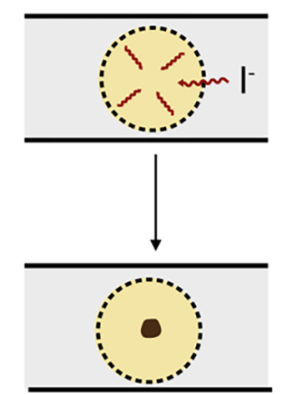

Slower segregation

\section{Pressure}

Figure 4. Schemes of the proposed mechanism to kinetically stabilize phase segregation by external physical pressure. (a) Light induces thermal expansion strain and decreases the energy barrier for halide migration by weakening the $\mathrm{Pb}-\mathrm{X}$ bonds. At high pressure, the compressive strain counteracts the light-induced strain and increases the activation energy for halide migration. (b) At ambient pressure, mainly the iodide ions move to the illuminated areas (dashed circle) to increase the volume of quickly formed iodide-rich islands, increasing their volume. At high pressure, halide ions move slower given the higher activation energy, leading to slower phase segregation.

in the dark also when $\mathrm{MA}^{+}$is partially replaced by a larger cation. In fact, as previously discussed, strain can also play a key role in the stability of mixed-cation mixed-halide perovskite compositions, which are reported to be less sensitive to lightinduced thermal expansion ${ }^{34}$ and phase segregation. ${ }^{20-22}$

Our measurements can also give insight into the mechanism of phase segregation. The formation rate of the low-energy iodide-rich phase appears to be faster than that of the highenergy bromide-rich phase for all the compositions and pressures explored. The total mixing ratio before light-soaking at ambient conditions calculated from the area and the energy position of the bleach is $0.531 \pm 0.001$ for $x=0.5$. Now that we know the $x$-values of the segregated phases calculated from the bleach energy positions and their relative peak areas, we can calculate whether the total mixing ratio has changed during the segregation. After $20 \mathrm{~min}$ of light-soaking, the mixing ratio is $0.46 \pm 0.01$, suggesting iodide enrichment of the illuminated area. Although most of the halide ions move within the illuminated area, the decrease in the total mixing ratio suggests that some iodide ions have moved from outside the illuminated region. At high pressure, a similar trend is observed $(x=0.47$ \pm 0.01 after light-soaking).
The similarity in total mixing ratio suggests that despite the changes in segregation rate and final composition, the total number of iodides moving into the probed area does not change with pressure. Furthermore, while both the segregation rate and the final composition of the iodide-rich phase are heavily affected by physical pressure, ${ }^{23}$ the bromide-rich phase does not show a clear trend with pressure. As an additional observation, the rate of evolution of the area of the bleach of the low-energy phase (roughly proportional to the volume fraction) is slower as compared to the rate obtained by the peak energy position in time, whereas the high-energy phase shows the same rate for the peak energy and peak area decay (see Figure S8). The rate of the evolution of the iodide peak area can be ascribed to two competing processes regulating the change in the area of the peak; on one hand, it increases due to a diffusion of iodide to the illuminated areas of the sample, and on the other hand, it decreases because of a reduction in the volume due to a loss of bromide species.

We hypothesize that all these observations could be explained by the formation of local, small iodide-rich islands (low-energy phase) with a well-defined composition from a small portion of the film that does not substantially affect the high-energy phase. Then, once those islands are formed, the bulk of the halides segregate via the migration of iodide ions into the initially formed islands, growing the volume of these domains. $^{38}$ It is important to note that the different rates extracted from the areas can also be the result of different dynamics before 15 ps for the low- and high-energy phases. Therefore, the interpretation is not trivial. In addition, we observe that during halide segregation, the total area of the two bleaches decreases over time (see Figure S9). The total area is constant at high pressure, again likely due to the halide immobilization. Finally, given that the halide migration likely occurs via a similar vacancy-mediated mechanism both in mixed- and single-halide compositions, we propose that unit cell size is equally important for halide migration in singlehalide systems and that a similar chemical approach as in mixed-halide compositions could be used to reduce the rate of halide migration in single-halide compositions, e.g., $\mathrm{MAPbI}_{3}$.

To conclude, we used pressure-dependent transient absorption to investigate the dynamics of phase segregation by tracking the iodide- and bromide-rich phase formation over time. We have shown that phase segregation in several mixedhalide $\mathrm{MAPb}\left(\mathrm{Br}_{x} \mathrm{I}_{1-x}\right)_{3} \quad(x=0.25,0.5$, and 0.7$)$ becomes substantially slower under pressure. We attribute the reduced segregation rate to a pressure-induced increase in the effective activation energy for the halide migration process. Firstprinciple calculations support this explanation. We show that reduction of unit cell volume achieved via partial or complete replacement of the $\mathrm{MA}^{+}$cation by the smaller $\mathrm{Cs}^{+}$cation results in a comparable slowing down of the phase segregation. Hence, this observation suggests the importance of the unit cell volume as one of the key factors for kinetic properties of ion migration and that chemically tuning the unit cell at ambient conditions (instead of applying physical pressure) may be used to kinetically suppress ion migration/phase segregation. While kinetic stabilization alone may not be a feasible route for long-term stability, ${ }^{39}$ these findings will help in understanding the key factors affecting the rate of halide migration and developing an effective strategy to suppress halide migration for the entire lifetime of the perovskite-based devices. 


\section{ASSOCIATED CONTENT}

\section{(s) Supporting Information}

The Supporting Information is available free of charge at https://pubs.acs.org/doi/10.1021/acsenergylett.0c01474.

$\Delta T / T$ scan as a function of time and probe energy for $x$ $=0.5$ without light-soaking at ambient pressure; $\Delta T / T$ traces of $x=0.5$ at different pressures after 2, 12, 32, 72, 97, and $197 \mathrm{~s}$; evolution of the low-energy iodide- and bromide-rich phases in real time for $x=0.25$ and 0.7 ; $\Delta T / T$ of $x=0.5$ after 1,2 , and 320 s fitted with single and double Gaussians; calculated energy barrier for $\mathrm{CsPbI}_{3}$ and $\mathrm{CsPbBr}_{3}$ as a function of the unit cell volume; evolution of the low-energy iodide- and bromide-rich phases in real time for $x=0.5$ in the case of the reduction of the unit cell volume by replacing $\mathrm{MA}^{+}$with $\mathrm{Cs}^{+}$; effective activation energies as a function of unit cell volume change induced by chemical engineering and physical pressure; evolution of the low-energy iodide- and bromide-rich phase peak areas in real time for $x=0.25,0.5$, and 0.7 and resulting segregation rates; decay of the total area as a function of pressure for $x=0.5$ and 0.7 ; derivation of relation between the segregation rate and the activation energy for ion migration; TID measurements and Arrhenius plot for $x=0.20$ in dark and light (PDF)

\section{AUTHOR INFORMATION}

\section{Corresponding Author}

Bruno Ehrler - Center for Nanophotonics, AMOLF, 1098 XG Amsterdam, The Netherlands; (1) orcid.org/0000-0002-53073241; Email: b.ehrler@amolf.nl

\section{Authors}

Loreta A. Muscarella - Center for Nanophotonics, AMOLF, 1098 XG Amsterdam, The Netherlands; 이이이.org/00000002-0559-4085

Eline M. Hutter - Center for Nanophotonics, AMOLF, 1098 XG Amsterdam, The Netherlands; Department of Chemistry, Utrecht University, 3584 CB Utrecht, The Netherlands; (1) orcid.org/0000-0002-5537-6545

Francesca Wittmann - Center for Nanophotonics, AMOLF, 1098 XG Amsterdam, The Netherlands

Young Won Woo - Department of Materials Science and Engineering, Yonsei University, Seoul 03722, Korea

Young-Kwang Jung - Department of Materials Science and Engineering, Yonsei University, Seoul 03722, Korea; (1) orcid.org/0000-0003-3848-8163

Lucie McGovern - Center for Nanophotonics, AMOLF, 1098 XG Amsterdam, The Netherlands; (o orcid.org/0000-00017263-5249

Jan Versluis - Center for Nanophotonics, AMOLF, 1098 XG Amsterdam, The Netherlands

Aron Walsh - Department of Materials Science and Engineering, Yonsei University, Seoul 03722, Korea; Department of Materials, Imperial College London, London SW7 2AZ, United Kingdom; O orcid.org/0000-0001-5460-7033

Huib J. Bakker - Center for Nanophotonics, AMOLF, 1098 XG Amsterdam, The Netherlands; O orcid.org/0000-0003-15645314

Complete contact information is available at: https://pubs.acs.org/10.1021/acsenergylett.0c01474

\section{Author Contributions}

L.A.M. performed the pressure-dependent TA experiments and data analysis together with E.M.H., under the supervision of B.E. F.W. assisted in the sample preparation, and J.V. assisted in the TA experiments under the supervision of H.J.B. L.McG. performed the TID experiment and the data analysis. Thermodynamic calculations were performed by A.W, Y.W.W., and Y.K.J. The manuscript was written by L.A.M. with input from all other authors.

\section{Notes}

The authors declare no competing financial interest.

\section{ACKNOWLEDGMENTS}

The work of L.A.M., E.M.H., F.W., L.McG., J.V., H.J.B., and B.E. is part of the Dutch Research Council (NWO) and was performed at the research institute AMOLF. The work of L.A.M. and L.McG. was supported by NWO Vidi grant 016.Vidi.179.005. The work of Y.W.W., Y.K.J., and A.W. was supported by the Creative Materials Discovery Program through the National Research Foundation of Korea (NRF) funded by Ministry of Science and ICT (2018M3D1A1058536). Y.W.W., Y.K.J., and A.W. are grateful to the UK Materials and Molecular Modelling Hub for computational resources, which is partially funded by EPSRC (EP/P020194/1).

\section{REFERENCES}

(1) Eperon, G. E.; Stranks, S. D.; Menelaou, C.; Johnston, M. B.; Herz, L. M.; Snaith, H. J. Formamidinium Lead Trihalide: A Broadly Tunable Perovskite for Efficient Planar Heterojunction Solar Cells. Energy Environ. Sci. 2014, 7 (3), 982-988.

(2) Zhang, F.; Zhong, H.; Chen, C.; Wu, X.; Hu, X.; Huang, H.; Han, J.; Zou, B.; Dong, Y. Brightly Luminescent and Color-Tunable Colloidal CH3NH3PbX3 (X = Br, I, Cl) Quantum Dots: Potential Alternatives for Display Technology. ACS Nano 2015, 9 (4), 45334542 .

(3) Adjokatse, S.; Fang, H. H.; Loi, M. A. Broadly Tunable Metal Halide Perovskites for Solid-State Light-Emission Applications. Mater. Today 2017, 20 (8), 413-424.

(4) McMeekin, D. P.; Sadoughi, G.; Rehman, W.; Eperon, G. E.; Saliba, M.; Hörantner, M. T.; Haghighirad, A.; Sakai, N.; Korte, L.; Rech, B.; et al. A Mixed-Cation Lead Mixed-Halide Perovskite Absorber for Tandem Solar Cells. Science 2016, 351 (6269), 151155.

(5) Christians, J. A.; Habisreutinger, S. N.; Berry, J. J.; Luther, J. M. Stability in Perovskite Photovoltaics: A Paradigm for Newfangled Technologies. ACS Energy Letters. 2018, 3 (9), 2136-2143.

(6) Hoke, E. T.; Slotcavage, D. J.; Dohner, E. R.; Bowring, A. R.; Karunadasa, H. I.; McGehee, M. D. Reversible Photo-Induced Trap Formation in Mixed-Halide Hybrid Perovskites for Photovoltaics. Chem. Sci. 2015, 6 (1), 613-617.

(7) Slotcavage, D. J.; Karunadasa, H. I.; McGehee, M. D. LightInduced Phase Segregation in Halide-Perovskite Absorbers. ACS Energy Letters. 2016, 1 (6), 1199-1205.

(8) Razera, R. A. Z.; Jacobs, D. A.; Fu, F.; Fiala, P.; Dussouillez, M.; Sahli, F.; Yang, T. C. J.; Ding, L.; Walter, A.; Feil, A. F.; et al. Instability of P-i-n Perovskite Solar Cells under Reverse Bias. J. Mater. Chem. A 2020, 8 (1), 242-250.

(9) Lee, H.; Gaiaschi, S.; Chapon, P.; Tondelier, D.; Bourée, J.-E.; Bonnassieux, Y.; Derycke, V.; Geffroy, B. Effect of Halide Ion Migration on the Electrical Properties of Methylammonium Lead TriIodide Perovskite Solar Cells. J. Phys. Chem. C 2019, 123 (29), 17728-17734.

(10) Futscher, M. H.; Lee, J. M.; McGovern, L.; Muscarella, L. A.; Wang, T.; Haider, M. I.; Fakharuddin, A.; Schmidt-Mende, L.; Ehrler, B. Quantification of Ion Migration in $\mathrm{CH} 3 \mathrm{NH} 3 \mathrm{PbI} 3$ Perovskite Solar 
Cells by Transient Capacitance Measurements. Mater. Horiz. 2019, 6 (7), 1497-1503.

(11) Yuan, H.; Debroye, E.; Janssen, K.; Naiki, H.; Steuwe, C.; Lu, G.; Moris, M.; Orgiu, E.; Uji-I, H.; De Schryver, F.; et al. Degradation of Methylammonium Lead Iodide Perovskite Structures through Light and Electron Beam Driven Ion Migration. J. Phys. Chem. Lett. 2016, 7 (3), 561-566.

(12) Elmelund, T.; Seger, B.; Kuno, M.; Kamat, P. V. How Interplay between Photo and Thermal Activation Dictates Halide Ion Segregation in Mixed Halide Perovskites. ACS Energy Lett. 2020, 5 (1), 56-63.

(13) Yoon, S. J.; Draguta, S.; Manser, J. S.; Sharia, O.; Schneider, W. F.; Kuno, M.; Kamat, P. V. Tracking Iodide and Bromide Ion Segregation in Mixed Halide Lead Perovskites during Photoirradiation. ACS Energy Lett. 2016, 1 (1), 290-296.

(14) Barker, A. J.; Sadhanala, A.; Deschler, F.; Gandini, M.; Senanayak, S. P.; Pearce, P. M.; Mosconi, E.; Pearson, A. J.; Wu, Y.; Srimath Kandada, A. R.; et al. Defect-Assisted Photoinduced Halide Segregation in Mixed-Halide Perovskite Thin Films. ACS Energy Lett. 2017, 2 (6), 1416-1424.

(15) Yang, X.; Yan, X.; Wang, W.; Zhu, X.; Li, H.; Ma, W.; Sheng, C. X. Light Induced Metastable Modification of Optical Properties in CH3NH3PbI3-XBrx Perovskite Films: Two-Step Mechanism. Org. Electron. 2016, 34, 79-83.

(16) Hu, M.; Bi, C.; Yuan, Y.; Bai, Y.; Huang, J. Stabilized Wide Bandgap MAPbBrxI3-x Perovskite by Enhanced Grain Size and Improved Crystallinity. Adv. Sci. 2016, 3 (6), 1500301.

(17) Ruth, A.; Brennan, M. C.; Draguta, S.; Morozov, Y. V.; Zhukovskyi, M.; Janko, B.; Zapol, P.; Kuno, M. Vacancy-Mediated Anion Photosegregation Kinetics in Mixed Halide Hybrid Perovskites: Coupled Kinetic Monte Carlo and Optical Measurements. ACS Energy Lett. 2018, 3 (10), 2321-2328.

(18) Knight, A. J.; Wright, A. D.; Patel, J. B.; McMeekin, D. P.; Snaith, H. J.; Johnston, M. B.; Herz, L. M. Electronic Traps and Phase Segregation in Lead Mixed-Halide Perovskite. ACS Energy Lett. 2019, 4 (1), 75-84.

(19) Beal, R. E.; Hagström, N. Z.; Barrier, J.; Gold-Parker, A.; Prasanna, R.; Bush, K. A.; Passarello, D.; Schelhas, L. T.; Brüning, K.; Tassone, C. J.; et al. Structural Origins of Light-Induced Phase Segregation in Organic-Inorganic Halide Perovskite Photovoltaic Materials. Matter 2020, 2 (1), 207-219.

(20) Dang, H. X.; Wang, K.; Ghasemi, M.; Tang, M. C.; De Bastiani, M.; Aydin, E.; Dauzon, E.; Barrit, D.; Peng, J.; Smilgies, D. M.; et al. Multi-Cation Synergy Suppresses Phase Segregation in Mixed-Halide Perovskites. Joule 2019, 3 (7), 1746-1764.

(21) Xu, J.; Boyd, C. C.; Yu, Z. J.; Palmstrom, A. F.; Witter, D. J.; Larson, B. W.; France, R. M.; Werner, J.; Harvey, S. P.; Wolf, E. J.; et al. Triple-Halide Wide-Band Gap Perovskites with Suppressed Phase Segregation for Efficient Tandems. Science 2020, 367 (6482), 1097-1104.

(22) Rehman, W.; McMeekin, D. P.; Patel, J. B.; Milot, R. L.; Johnston, M. B.; Snaith, H. J.; Herz, L. M. Photovoltaic Mixed-Cation Lead Mixed-Halide Perovskites: Links between Crystallinity, PhotoStability and Electronic Properties. Energy Environ. Sci. 2017, 10 (1), 361-369.

(23) Hutter, E. M.; Muscarella, L. A.; Wittmann, F.; Versluis, J.; McGovern, L.; Bakker, H. J.; Woo, Y.-W.; Jung, Y.-K.; Walsh, A.; Ehrler, B. Thermodynamic Stabilization of Mixed-Halide Perovskites against Phase Segregation. Cell Reports Phys. Sci. 2020, 1 (8), 100120.

(24) Hopper, T. R.; Gorodetsky, A.; Frost, J. M.; Muller, C.; Lovrincic, R.; Bakulin, A. A. Ultrafast Intraband Spectroscopy of HotCarrier Cooling in Lead-Halide Perovskites. ACS Energy Lett. 2018, 3 (9), 2199-2205.

(25) Yoon, S. J.; Kuno, M.; Kamat, P. V. Shift Happens. How Halide Ion Defects Influence Photoinduced Segregation in Mixed Halide Perovskites. ACS Energy Lett. 2017, 2 (7), 1507-1514.

(26) Futscher, M. H.; Gangishetty, M. K.; Congreve, D. N.; Ehrler, B. Quantifying Mobile Ions and Electronic Defects in PerovskiteBased Devices with Temperature-Dependent Capacitance Measure- ments: Frequency vs Time Domain. J. Chem. Phys. 2020, 152 (4), 044202.

(27) Futscher, M. H.; Lee, J. M.; McGovern, L.; Muscarella, L. A.; Wang, T.; Haider, M. I.; Fakharuddin, A.; Schmidt-Mende, L.; Ehrler, B. Quantification of Ion Migration in $\mathrm{CH} 3 \mathrm{NH} 3 \mathrm{PbI} 3$ Perovskite Solar Cells by Transient Capacitance Measurements. Mater. Horiz. 2019, 6 (7), 1497-1503.

(28) Groeneveld, B. G. H. M.; Adjokatse, S.; Nazarenko, O.; Fang, H. H.; Blake, G. R.; Portale, G.; Duim, H.; ten Brink, G. H.; Kovalenko, M. V.; Loi, M. A. Stable Cesium Formamidinium Lead Halide Perovskites: A Comparison of Photophysics and Phase Purity in Thin Films and Single Crystals. Energy Technol. 2020, 8 (4), 1901041.

(29) Ghosh, D.; Acharya, D.; Zhou, L.; Nie, W.; Prezhdo, O. V.; Tretiak, S.; Neukirch, A. J. Lattice Expansion in Hybrid Perovskites: Effect on Optoelectronic Properties and Charge Carrier Dynamics. J. Phys. Chem. Lett. 2019, 10 (17), 5000-5007.

(30) Zhao, J.; Deng, Y.; Wei, H.; Zheng, X.; Yu, Z.; Shao, Y.; Shield, J. E.; Huang, J. Strained Hybrid Perovskite Thin Films and Their Impact on the Intrinsic Stability of Perovskite Solar Cells. Sci. Adv. 2017, 3 (11), No. eaao5616.

(31) Zhao, Y. C.; Zhou, W. K.; Zhou, X.; Liu, K. H.; Yu, D. P.; Zhao, Q. Quantification of Light-Enhanced Ionic Transport in Lead Iodide Perovskite Thin Films and Its Solar Cell Applications. Light: Sci. Appl. 2017, 6 (5), No. e16243.

(32) Lee, J. W.; Kim, S. G.; Yang, J. M.; Yang, Y.; Park, N. G. Verification and Mitigation of Ion Migration in Perovskite Solar Cells. APL Mater. 2019, 7 (4), 041111.

(33) Rolston, N.; Bennett-Kennett, R.; Schelhas, L. T.; Luther, J. M.; Christians, J. A.; Berry, J. J.; Dauskardt, R. H. Comment on "LightInduced Lattice Expansion Leads to High-Efficiency Perovskite Solar Cells. Science 2020, 368 (6488), eaay8691.

(34) Rolston, N.; Bennett-Kennett, R.; Schelhas, L. T.; Luther, J. M.; Christians, J. A.; Berry, J. J.; Dauskardt, R. H. Comment on "LightInduced Lattice Expansion Leads to High-Efficiency Perovskite Solar Cells. Science 2020, 368 (6488), No. eaay8691.

(35) Xue, D. J.; Hou, Y.; Liu, S. C.; Wei, M.; Chen, B.; Huang, Z.; Li, Z.; Sun, B.; Proppe, A. H.; Dong, Y.; et al. Regulating Strain in Perovskite Thin Films through Charge-Transport Layers. Nat. Commun. 2020, 11 (1), 1514.

(36) Mathew, P. S.; Samu, G. F.; Janáky, C.; Kamat, P. V. Iodine (I) Expulsion at Photoirradiated Mixed Halide Perovskite Interface. Should I Stay or Should I Go? ACS Energy Lett. 2020, 5 (6), 18721880.

(37) Ferdani, D. W.; Pering, S. R.; Ghosh, D.; Kubiak, P.; Walker, A. B.; Lewis, S. E.; Johnson, A. L.; Baker, P. J.; Islam, M. S.; Cameron, P. J. Partial Cation Substitution Reduces Iodide Ion Transport in Lead Iodide Perovskite Solar Cells. Energy Environ. Sci. 2019, 12 (7), 2264-2272.

(38) Draguta, S.; Sharia, O.; Yoon, S. J.; Brennan, M. C.; Morozov, Y. V.; Manser, J. M.; Kamat, P. V.; Schneider, W. F.; Kuno, M. Rationalizing the Light-Induced Phase Separation of Mixed Halide Organic-Inorganic Perovskites. Nat. Commun. 2017, 8 (1), 200.

(39) Ehrler, B.; Hutter, E. M. Routes toward Long-Term Stability of Mixed-Halide Perovskites. Matter 2020, 2 (4), 800-802. 